\title{
Risk Factors for Pregnancy Complications and Postpartum Glucose Intolerance in Women With Gestational Diabetes Mellitus
}

Naohisa Masuko

Kobe University

Kenji Tanimura

Kobe University

Nobue Kojima

Kobe University

Hitomi Imafuku

Kobe University

Masashi Deguchi

Kobe University

Kazumichi Fujioka

Kobe University

Yuko Okada

Kobe University

Yushi Hirota

Kobe University

Wataru Ogawa

Kobe University

Hideto Yamada ( $\nabla$ yhideto@med.kobe-u.ac.jp )

Kobe University

\section{Research Article}

Keywords: GDM, GI, OGTT, HDP, postpartum

Posted Date: December 9th, 2020

DOl: https://doi.org/10.21203/rs.3.rs-116971/v1

License: (c) (i) This work is licensed under a Creative Commons Attribution 4.0 International License.

Read Full License 


\section{Abstract}

This prospective cohort study aimed to evaluate the risk factors for pregnancy complications and postpartum glucose intolerance (GI) in women with gestational diabetes mellitus (GDM). A total of 140 women with GDM were enrolled. Of these, 115 underwent a 75-g oral glucose tolerance test (OGTT) at 12 weeks after delivery. Clinical factors and parameters in the antepartum 75-g OGTT associated with pregnancy complications and postpartum GI were evaluated. Women with GDM experienced pregnancy complications, including hypertensive disorders of pregnancy (HDP, $n=19)$, preterm delivery (PD, $n=17)$, heavy-for-date (HFD, $n=19)$, and light-for-date (LFD, $n=12)$, and 22 of the 115 women with GDM developed postpartum $\mathrm{GI}$. The univariate and multivariable logistic regression analyses revealed the following risk factors: histories of hypertension (odds ratio [OR], 23.8; 95\% confidence interval [CI], 4.2-134.7; $p<0.01$ ) for HDP; histories of hypertension (OR, 9.8; 95\% Cl, 2.5-38.9; $p<0.01)$ for PD; HbA1c levels (OR, 7.6; $95 \%$ $\mathrm{Cl}, 1.5-37.9 ; p<0.05)$ for HFD; and oral deposition index (DI) (OR, $0.1 ; 95 \% \mathrm{Cl}, 0.02-0.7 ; p<0.01)$ for postpartum GI. Higher HbA1c levels and lower oral DI on the antepartum 75-g OGTT may be useful markers for identifying GDM women who are at high risk for HFD and postpartum Gl, respectively.

\section{Introduction}

Pregnant women with gestational diabetes mellitus (GDM) have an increased risk of pregnancy and neonatal complications, including hypertensive disorders of pregnancy (HDP), preterm delivery (PD), polyhydramnios, shoulder dystocia, macrosomia, heavy-for-date (HFD) newborn, light-for-date (LFD) newborn, and neonatal hypoglycemia ${ }^{1-6}$.

Previous studies have evaluated associations between maternal clinical or laboratory findings of antepartum 75-g oral glucose tolerance test (OGTT) and pregnancy complications in women with GDM. PD and HDP are associated with normal fasting and elevated post-load blood glucose (BG) levels ${ }^{6}$. HFD is associated with elevated fasting and normal post-load BG levels $s^{6,7}$ and post-load hyperglycemia ${ }^{8}$. Moreover, macrosomia is associated with post-load hyperglycemia ${ }^{8}$, fasting hyperglycemia, and excessive gestational weight gain ${ }^{7,9}$.

A systematic review and meta-analysis has demonstrated that women with GDM have a 7.4-fold increased risk of developing type 2 diabetes mellitus after delivery compared with those without GDM ${ }^{10}$. We have reported that, in women with GDM, the low insulinogenic index (II) levels on the antepartum 75-g OGTT is a risk factor for developing glucose intolerance (GI) during the early postpartum period ${ }^{11}$.

This prospective cohort study aimed to assess predictive clinical factors and laboratory parameters in the antepartum 75-g OGTT for pregnancy complications and GI during the early postpartum period among women with GDM.

\section{Results}


Of 3,494 pregnant women who had singleton deliveries at the Kobe University Hospital, 140 (4.0\%) were diagnosed with GDM from January 2011 to December 2018. The indications for the antepartum 75-g OGTT in the 140 pregnant women with GDM were as follows: a 1-hr BG level on a 50-g glucose challenge tests $\geq 140 \mathrm{mg} / \mathrm{dL}$ ( $n=99$ ); casual BG level $\geq 100 \mathrm{mg} / \mathrm{dL}$ ( $n=13$ ); suspicion of HFD and/or polyhydramnios on ultrasound examinations during pregnancy $(n=10)$; and presence of other risk factors of GDM, including a history of GDM, obesity, and persistent glycosuria $(n=18)$. Twenty-five of the 140 women with GDM refused to receive a 75-g OGTT at 12 weeks after delivery. Therefore, 115 of the 140 (82.1\%) women with GDM were included in the analyses of risks for GI during the early postpartum period.

\section{Clinical factors and parameters in the antepartum 75-g OGTT associated with the occurrence of HDP in women with GDM}

Nineteen of the 140 (13.6\%) pregnant women with GDM had HDP. Table 1 shows the clinical characteristics of participants and result of logistic regression analyses of factors associated with HDP. The group of GDM women with HDP (HDP group) had a significantly higher body mass index (BMI) prior to pregnancy $(p<0.05)$ and the frequency of a history of hypertension $(p<0.01)$ than the group without HDP (non-HDP group). Furthermore, the HDP group had a significantly less weight gain during pregnancy $(p<0.01)$ than the non-HDP group. No significant differences were observed in any parameter in the antepartum 75-g OGTT between the two groups.

Univariate logistic regression analyses demonstrated that the BMI prior to pregnancy (odds ratio [OR], 1.1; $95 \%$ confidence interval $[\mathrm{Cl}], 1.0-1.2 ; p<0.05)$, weight gain during pregnancy $(\mathrm{OR}, 0.8 ; 95 \% \mathrm{Cl}, 0.8-0.9$; $p<0.01)$, frequency of the presence of a history of hypertension ( $\mathrm{OR}, 43.3 ; 95 \% \mathrm{Cl}, 8.2-229.4 ; p<0.01)$, and 1 -hr BG levels (OR, 1.0; $95 \% \mathrm{Cl}, 0.96-1.0 ; p<0.05)$ were associated with the occurrence of HDP in women with GDM. Multivariable logistic regression analyses of the three factors revealed that the presence of a history of hypertension (OR, 23.8; $95 \% \mathrm{Cl}, 4.2-134.7 ; p<0.01)$ was a single independent factor associated with HDP.

\section{Clinical factors and parameters in the antepartum 75-g OGTT associated with the occurrence of preterm delivery at 36 or less gestational weeks (GW) in women with GDM}

Seventeen of the $140(12.1 \%)$ pregnant women with GDM had PD at 36 or less GW. Eleven of the 17 $(64.7 \%)$ pregnancies resulted in preterm births following a threatened premature labor, and the remaining $6(35.3 \%)$ resulted in medically indicated preterm births ( 5 for HDP; 1 for exacerbations of underlying diseases).

Table 2 shows the clinical characteristics of participants and result of logistic regression analyses of factors associated with PD. The group of GDM women with PD (PD group) had a significantly higher parity $(p<0.05)$ and frequency of the presence of a history of hypertension $(p<0.01)$ than the group without PD (non-PD group). 
Univariate logistic regression analyses demonstrated that the presence of a history of hypertension (OR, $9.8 ; 95 \% \mathrm{Cl}, 2.5-38.9 ; p<0.01)$ was a single independent factor associated with $\mathrm{PD}$ in pregnant women with GDM.

\section{Clinical factors and parameters in the antepartum 75-g OGTT associated with the occurrence of HFD infants in women with GDM}

Nineteen of the 140 (13.6\%) women with GDM had HFD newborns. Table 3 reveals the clinical characteristics of participants and results of logistic regression analyses of factors associated with HFD. The group of GDM women with HFD (HFD group) had a significantly higher weight gain during pregnancy $(p<0.05)$ and HbA1c levels $(p<0.05)$ than the group without HFD (non-HFD group).

Univariate logistic regression analyses demonstrated that $\mathrm{HbA} 1 \mathrm{c}$ levels $(\mathrm{OR}, 7.6 ; 95 \% \mathrm{Cl}, 1.5-37.9$; $p<0.05)$ was an independent factor associated with HFD.

\section{Clinical factors and parameters in the antepartum 75-g OGTT associated with the occurrence of LFD infants in women with GDM}

Twelve of the 140 (8.6\%) pregnant women with GDM had LFD newborns. Table 4 exhibits the clinical characteristics of participants and result of logistic regression analyses of factors associated with LFD. The group of GDM women with LFD (LFD group) had a significantly lower HbA1c levels $(p<0.05)$ than the group without LFD (non-LFD group).

No clinical factors and parameters in the antepartum 75-g OGTT were selected as factors associated with LFD in pregnant women with GDM by univariate logistic regression analyses.

\section{Clinical factors and parameters in the antepartum 75-g OGTT associated with the occurrence of postpartum GI in women with GDM}

Twenty-two of the 115 (19.1\%) pregnant women with GDM had GI at 12 weeks after delivery, including one, two, and 19 women with diabetes mellitus (DM), impaired fasting glucose (IFG), and impaired glucose tolerance (IGT), respectively.

Table 5 shows the clinical characteristics of participants and results of logistic regression analyses of factors associated with GI during the early postpartum period. The group of GDM women with postpartum $\mathrm{GI}$ (GI group) had a significantly lower oral DI $(p<0.01)$ than the group without postpartum $\mathrm{GI}$ (non-Gl group).

Univariate logistic regression analyses demonstrated that fasting BG (FBG) (OR, 1.0; 95\% $\mathrm{Cl}, 1.0-1.1$; $p<0.05)$ and oral disposition index (DI) $(\mathrm{OR}, 0.1 ; 95 \% \mathrm{Cl}, 0.03-0.5 ; p<0.01)$ were associated with the occurrence of GI during the early postpartum period in women with GDM. Multivariable logistic regression analyses of the two factors revealed that oral $\mathrm{DI}(\mathrm{OR}, 0.1 ; 95 \% \mathrm{Cl}, 0.02-0.7 ; p<0.01)$ was an independent factor associated with $\mathrm{GI}$ during the early postpartum period. 


\section{Discussion}

This study used the International Association of Diabetes and Pregnancy Study Groups (IADPSG) criteria for diagnosing $\mathrm{GDM}^{12}$, and 140 of the 3,494 (4.0\%) pregnant women who had singleton deliveries were diagnosed with GDM. Because medians of the prevalence of GDM in Japan were reported to be $2.8 \%-$ $13.0 \%{ }^{13}$, the prevalence of GDM in this study was thought to be valid. The incidences of HDP, PD, HFD newborns, and LFD newborns among women with GDM were $13.6 \%, 12.1 \%, 13.6 \%$, and $8.6 \%$, respectively. The incidences of pregnancy complications in women with GDM that were diagnosed based on the IADPSG criteria have been reported as follows: HDP, $8.2 \%{ }^{14}$; PD, $6.5 \%-8.4 \%{ }^{15,16}$; large-for-gestational-age (LGA) newborns, 9.1\%-21.4\% ${ }^{15,16}$; and small-for-gestational-age (SGA) newborns, $7.6 \%-8.0 \% 16,17$. Although the present study had higher incidences of HDP and PD than the previous studies, the incidence $(19.1 \%)$ of GI during the early postpartum period in women with GDM was comparable to those $(16.7 \%-$ $36.6 \%$ in previous studies ${ }^{11,18,19}$. Our study had higher incidences of HDP and PD than the previous studies because Kobe University Hospital has a maternal-fetal center where pregnant women with HDP and threatened premature labor are often referred from other hospitals and clinics.

For the first time, this prospective cohort study of pregnant women with GDM simultaneously assessed both the clinical factors and parameters in the antepartum 75-g OGTT for pregnancy complications and $\mathrm{Gl}$ at 12 weeks after delivery by logistic regression analyses using a stepwise approach, and revealed the following risk factors: the presence of histories of hypertension both for HDP and PD at 36 or less GW; higher HbA1c levels for HFD infants; and lower oral DI for postpartum GI.

Obesity, chronic hypertension, and a history of HDP have been reported as major risk factors for HDP 20,21 . Our result is comparable with those in previous studies.

Previous prospective cohort studies, including a general population of pregnant women, revealed that older maternal age, lower $\mathrm{BMI}$, and GDM were risk factors for $\mathrm{PD}^{22,23}$. When subjects were limited to women with GDM as in our study, not maternal age and BMI prior to pregnancy, but a history of hypertension was selected as a risk factor for PD. In addition, we found that a history of hypertension was an independent risk factor for HDP in women with GDM. It is likely that pregnancies of GDM women with histories of hypertension result in preterm births due to recurrent HDP.

Several retrospective studies demonstrated that elevated fasting BG levels were associated with HFD ${ }^{6,7}$, and others suggested that post-load hyperglycemia was associated with $\mathrm{HFD}^{8}$. In this prospective cohort study, the univariate and multivariable logistic regression analyses demonstrated that the higher $\mathrm{HbA} 1 \mathrm{c}$ level was a risk factor for HFD in pregnant women with GDM. This result indicated that HFD in women with GDM was more closely associated with continuous hyperglycemia, which higher HbA1c levels reflect, rather than higher levels of fasting or post-load BG on the antepartum 75-g OGTT.

The present study also found neither differences in clinical factors and parameters in the antepartum 75$g$ OGTT between the LFD and non-LFD groups nor any factors associated with the occurrence of LFD in 
pregnant women with GDM. SGA/LFD newborns are likely to be associated with severe $\mathrm{DM}^{7,24}$ rather than GDM.

Univariate and multivariable logistic regression analyses revealed for the first time that oral DI was an independent risk factor for postpartum Gl in pregnant women with GDM. Previous retrospective studies demonstrated that low II and II/fasting IRI were associated with postpartum GI in patients with GDM ${ }^{18,25}$. A previous prospective cohort study of 72 pregnant women with GDM revealed that a low II in the antepartum 75-g OGTT is an independent risk factor for developing GI during the early postpartum period $^{11}$. It was reported that among the Japanese-American adults, including males and non-pregnant women, the low oral DI was predictive of developing DM in the future ${ }^{26}$. DI represents a hyperbolic relationship between insulin secretion and insulin sensitivity ${ }^{27,28}$. Therefore, this parameter represents the insulin secretory capacity of pancreatic $\beta$ cells adjusted for insulin sensitivity ${ }^{26}$. An adequate insulin secretory response of pancreatic $\beta$ cells adapting to changes in insulin sensitivity might be significant for the maintenance of normal glucose tolerance during the postpartum period. Pregnant women with low oral DI on the antepartum 75-g OGTT are at high risk not only for GI during the early postpartum period, but also for DM in the future.

This prospective cohort study demonstrated that a history of hypertension was a risk factor for HDP and $\mathrm{PD}$ in pregnant women with GDM, and higher $\mathrm{HbA1c}$ levels was a risk factor for HFD newborns. A low oral DI on the antepartum 75-g OGTT was an independent risk factor for GI during the early postpartum period in women with GDM. These findings may enable clinicians to effectively identify and manage women with GDM who are at high risks for pregnancy complications and DM in the future.

\section{Methods}

\section{Study design and participants}

This prospective cohort study enrolled women with singleton pregnancies who were diagnosed with GDM by the 75-g OGTT during pregnancy and delivered at the Kobe University Hospital from January 2011 to December 2018. The study was approved by the Institutional Review Board of the Kobe University Hospital (reference number 200228), and informed consent was obtained from all participants. All research was performed in accordance with the relevant guidelines and regulations.

\section{Procedures}

All pregnant women who visited or were referred to the Kobe University Hospital underwent screening for GDM both at 10-14 and 24-28 GW. Pregnant women who had casual BG levels of $\geq 100 \mathrm{mg} / \mathrm{dL}$ $(5.5 \mathrm{mmol} / \mathrm{L})$ at $10-14 \mathrm{GW}$, or those who had 1 -hr BG levels of $\geq 140 \mathrm{mg} / \mathrm{dL}(7.8 \mathrm{mmol} / \mathrm{L})$ on $50-\mathrm{g}$ glucose challenge tests (GCT) at 24-28 GW, or those with risk factors for GDM, including obesity, family history of DM , past history of macrosomia, presence of persistent glycosuria, polyhydramnios, and suspected HFD, underwent the 75-g OGTT. According to the IADPSG criteria ${ }^{12}$, the diagnosis of GDM is 
made when any of the following are met: FBG $\geq 92 \mathrm{mg} / \mathrm{dL}(5.1 \mathrm{mmol} / \mathrm{L}), 1-\mathrm{hr} \mathrm{BG} \geq 180 \mathrm{mg} / \mathrm{dL}$ $(10.0 \mathrm{mmol} / \mathrm{L})$, or $2-\mathrm{hr} B G \geq 153 \mathrm{mg} / \mathrm{dL}$ ( $8.5 \mathrm{mmol} / \mathrm{L})$. BG and immunoreactive insulin (IRI) levels at 0 , $0.5,1,1.5$, and $2 \mathrm{hr}$ after the oral ingestion of $75-\mathrm{g}$ glucose were also measured, and the total area under the curve (AUC) of glucose and insulin were calculated by the trapezoid method.

As an insulin resistance parameter, the homeostasis model assessment-insulin resistance (HOMA-IR) $(=\mathrm{FBG}(\mathrm{mg} / \mathrm{dL}) \times$ fasting IRI $(\mathrm{FIRI})(\mu \mathrm{U} / \mathrm{mL}) / 405)$ was used. HOMA $\beta(=360 \times \mathrm{FIRI}(\mu \mathrm{U} / \mathrm{mL}) /[\mathrm{FBG}$ $(\mathrm{mg} / \mathrm{dL})-63])$ and insulinogenic index (II) $(=[0.5-\mathrm{hr}$ IRI $(\mu \mathrm{U} / \mathrm{mL})-\mathrm{FIRI}(\mu \mathrm{U} / \mathrm{mL})] /[0.5-\mathrm{hr} \mathrm{BG}(\mathrm{mg} / \mathrm{dL})-\mathrm{FBG}$ $(\mathrm{mg} / \mathrm{dL})])$ were calculated for evaluating the insulin secretory capacity of pancreatic $\beta$ cells. The oral $\mathrm{DI}$, which represents the compensation of pancreatic $\beta$ cells for insulin resistance, was calculated as the product of the Matsuda index of insulin sensitivity and the ratio of the AUC of insulin to the AUC of glucose during the $\mathrm{OGTT}^{27}$. The Matsuda index was calculated using the following formula: $10^{4} / \sqrt{ }$ (FGB $\times$ FIRI × mean BG during 75-g OGTT × mean IRI during 75-g OGTT) ${ }^{29}$.

All pregnant women diagnosed with GDM were referred to diabetologists in the Kobe University Hospital and underwent self-monitoring of blood glucose (SMBG) and diet therapy. If FBG levels exceeded $100 \mathrm{mg} / \mathrm{dL}$, or 2-hr BG levels exceeded $120 \mathrm{mg} / \mathrm{dL}$ in SMBG regardless of diet therapy, an insulin therapy was started. Insulin doses were adjusted to achieve both FBG levels of $<100 \mathrm{mg} / \mathrm{dL}$ and 2-hr BG levels of $<120 \mathrm{mg} / \mathrm{dL}$.

All pregnant women with GDM were instructed to undergo a 75-g OGTT at 12 weeks after delivery. Using the WHO's 1999 criteria $^{30}$, DM was diagnosed by either FBG levels of $\geq 126 \mathrm{mg} / \mathrm{dL}(7.0 \mathrm{mmol} / \mathrm{L})$ or 2-hr BG levels of $\geq 200 \mathrm{mg} / \mathrm{dL}$ ( $11.1 \mathrm{mmol} / \mathrm{L}$ ). IFG was diagnosed by FBG levels of $\geq 110 \mathrm{mg} / \mathrm{dL}$ ( $6.1 \mathrm{mmol} / \mathrm{L})$, and IGT was diagnosed by 2 -hr BG levels of $\geq 140 \mathrm{mg} / \mathrm{dL}(7.8 \mathrm{mmol} / \mathrm{L})$. GI was defined by the presence of DM, IFG, or IGT. FBG levels of $<110 \mathrm{mg} / \mathrm{dL}(6.1 \mathrm{mmol} / \mathrm{L})$ and 2-hr BG levels of $<140 \mathrm{mg} / \mathrm{dL}(7.8 \mathrm{mmol} / \mathrm{L})$ were identified as normal.

This study assessed pregnancy complications, including HDP, PD at 36 or less GW, and HFD or LFD newborns. HFD and LFD were defined as newborns with a birth weight $>90$ th and $<10$ th percentile for gestational age, respectively.

\section{Statistical analysis}

Clinical characteristics were compared between pregnancies with each pregnancy complication or with GI during the early postpartum period and pregnancies without them. Differences between the two groups were analyzed using the Mann-Whitney $U$ test, Fisher exact test, and $\chi 2$ test. $P$ values $<0.05$ were considered statistically significant. The stepwise approach was used to evaluate clinical factors and parameters in the antepartum 75-g OGTT associated with each pregnancy complication and GI during the early postpartum period. Variables with $P$ values $<0.05$ in univariate logistic regression analyses were subjected to multivariable logistic regression analyses, and variables with $P$ values $<0.05$ in multivariable logistic regression analyses were determined as clinical factors and parameters in the antepartum 75-g OGTT associated with each pregnancy complication or GI during the early postpartum period in women 
with GDM. All statistical analyses were performed using the SPSS software, version 19 (SPSS Inc., Chicago, Illinois).

\section{Declarations}

\section{Data availability}

The datasets analyzed during the current study are available from the corresponding authors upon a reasonable request.

\section{Author contributions statement}

N.M. performed statistical analysis and made a draft of the manuscript. K.T. helped to analyze the data and write the manuscript. N.K., H.I., M.D., K.F., Y.O., Y.H., and W.O. collected the data and supervised the study. H.Y. designed the study and revised critically the manuscript. All authors read and approved the final manuscript.

\section{Competing interests}

The authors declare no competing interest.

\section{References}

1. Landon, M. B. et al. The relationship between maternal glycemia and perinatal outcome. Obstet. Gynecol.117, 218-224 (2011).

2. Aktün, H. L., Uyan, D., Yorgunlar, B. \& Acet, M. Gestational diabetes mellitus screening and outcomes. J. Turkish Ger. Gynecol. Assoc.16, 25-29 (2015).

3. Scifres, C. M. et al. Large-for-gestational-age ultrasound diagnosis and risk for cesarean delivery in women with gestational diabetes mellitus. Obstet. Gynecol.126, 978-986 (2015).

4. Rosen, H. et al. Delivery outcomes of large-for-gestational-age newborns stratified by the presence or absence of gestational diabetes mellitus. Int. J. Gynecol. Obstet.141, 120-125 (2018).

5. Crowther, Caroline A.; Hiller, Janet E.; Moss, John R.; McPhee, Andrew J.; Jeffries, William S.; Robinson, J. S. Effect of treatment of gestational diabetes mellitus on pregnancy outcomes. N Eng/ J Med352, 2477-2486 (2005).

6. Black, M. H., Sacks, D. A., Xiang, A. H. \& Lawrence, J. M. Clinical outcomes of pregnancies complicated by mild gestational diabetes mellitus differ by combinations of abnormal oral glucose tolerance test values. Diabetes Care33, 2524-2530 (2010).

7. Feng, H. et al. Relationship between oral glucose tolerance test characteristics and adverse pregnancy outcomes among women with gestational diabetes mellitus. Chin. Med. J. (Engl).130, 1012-1018 (2017). 
8. Combs, C. A., Gunderson, E., Kitzmiller, J. L., Gavin, L. A. \& Main, E. K. Relationship of fetal macrosomia to maternal postprandial glucose control during pregnancy. Diabetes Care15, 12511257 (1992).

9. Egan, A. M. et al. ATLANTIC-DIP: Excessive gestational weight gain and pregnancy outcomes in women with gestational or pregestational diabetes mellitus. J. Clin. Endocrinol. Metab.99, 212-219 (2014).

10. Bellamy, L., Casas, J. P., Hingorani, A. D. \& Williams, D. Type 2 diabetes mellitus after gestational diabetes: a systematic review and meta-analysis. Lancet373, 1773-1779 (2009).

11. Kojima, N. et al. Risk factors for postpartum glucose intolerance in women with gestational diabetes mellitus. Gynecol. Endocrinol.32, 803-806 (2016).

12. Metzger, B. E. International Association of Diabetes and Pregnancy Study Groups recommendations on the diagnosis and classification of hyperglycemia in pregnancy. Diabetes Care33, 676-682 (2010).

13. Nguyen, C. L., Pham, N. M., Binns, C. W., Van Duong, D. \& Lee, A. H. Prevalence of gestational diabetes mellitus in eastern and southeastern Asia: A systematic review and meta-analysis. J. Diabetes Res.2018, doi: 10.1155/2018/6536974 (2018).

14. Ethridge, J. K., Catalano, P. M. \& Waters, T. P. Perinatal outcomes associated with the diagnosis of gestational diabetes made by the international association of the diabetes and pregnancy study groups criteria. Obstet. Gynecol.124, 571-578 (2014).

15. Bodmer-Roy, S., Lucie, M., Jocelyne, C. \& Evelyne, R. Pregnancy outcomes in women with and without gestational diabetes mellitus according to the international association of the diabetes and pregnancy study groups criteria. Obstet. Gynecol.120, 746-752 (2012).

16. Kim, M. H. et al. Pregnancy outcomes of women additionally diagnosed as gestational diabetes by the International Association of the Diabetes and Pregnancy Study groups criteria. Diabetes Metab. J.43, 766-775 (2019).

17. Shindo, R., Aoki, S., Kasai, J., Saigusa, Y. \& Nakanishi, SayuriMiyagi, E. Have pregnancy outcomes improved with the introduction of the International Association of Diabetes and Pregnancy Study Groups criteria in Japan? J. Diabetes Investig.67, 15-20 (2020).

18. Kondo, M., Nagao, Y., Mahbub, M. H., Tanabe, T. \& Tanizawa, Y. Factors predicting early postpartum glucose intolerance in Japanese women with gestational diabetes mellitus: decision-curve analysis. Diabet. Med.35, 1111-1117 (2018).

19. Inoue, S. et al. Predictors of abnormal glucose tolerance in the early postpartum period in patients with gestational diabetes. Kurume Med. J.62, 47-51 (2015).

20. Rey, E. \& Couturier, A. The prognosis of pregnancy in women with chronic hypertension. Am. J. Obstet. Gynecol.171, 410-416 (1994).

21. Zhang, J., Troendle, J. F. \& Levine, R. J. Risks of hypertensive disorders in the second pregnancy. Paediatr. Perinat. Epidemiol.15, 226-231 (2001). 
22. Tamura, N. et al. Different risk factors for very low birth weight, term-small-for-gestational-age, or preterm Birth in Japan. Int. J. Environ. Res. Public Health15, doi: 10.3390/ijerph15020369 (2018).

23. Tellapragada, C. et al. Risk factors for preterm birth and low birth weight among pregnant Indian women: A hospital-based prospective study. J. Prev. Med. Public Heal.49, 165-175 (2016).

24. Eriksson, U. J., Cederberg, J. \& Wentzel, P. Congenital malformations in offspring of diabetic mothers - Animal and human studies. Rev. Endocr. Metab. Dis.4, 79-93 (2003).

25. Kugishima, Y. et al. Risk factors associated with abnormal glucose tolerance in the early postpartum period among Japanese women with gestational diabetes. Int. J. Gynecol. Obstet.129, 42-45 (2015).

26. Utzschneider, K. M. et al. Oral Disposition index predicts the development of future diabetes above and beyond fasting and 2-h glucose levels. Diabetes Care32, 335-341 (2009).

27. Retnakaran, R. et al. Hyperbolic relationship between insulin secretion and sensitivity on oral glucose tolerance test. Obesity16, 1901-1907 (2008).

28. Bergman, R. N., Ader, M., Huecking, K. \& Citters, G. Van. Accurate assessment of beta-cell function: The hyperbolic correction. 51, S212-S220 (2002).

29. M, Matsuda RA, D. Insulin sensitivity indices obtained from oral glucose tolerance testing: comparison with the euglycemic insulin clamp. Diabetes Care22, 1462-1470 (1999).

30. World Health Organization. Definition, diagnosis and classification of diabetes mellitus and its complications, Part 1: Diagnosis and classification of diabetes mellitus: Report of the WHO Consultation. Geneva: World Health Organization. (WHO/NCD/NCS/99.2). World Health59, (1999).

\section{Tables}

Due to technical limitations, table $1,2,3,4,5$ is only available as a download in the Supplemental Files section.

\section{Supplementary Files}

This is a list of supplementary files associated with this preprint. Click to download.

- SciRepMasukoetal.Table1.xlsx

- SciRepMasukoetal.Table1.xlsx

- SciRepMasukoetal.Table2.xlsx

- SciRepMasukoetal.Table2.xlsx

- SciRepMasukoetal.Table3.xlsx

- SciRepMasukoetal.Table3.xlsx

- SciRepMasukoetal.Table4.xlsx

- SciRepMasukoetal.Table4.xlsx

- SciRepMasukoetal.Table5.xlsx 
- SciRepMasukoetal.Table5.xIsx

Page $11 / 11$ 